\title{
Evaluación citológica en sala durante las biopsias de lesiones pancreáticas sólidas guiadas por ultrasonido endoscópico
}

\author{
Rapid on-site cytologic evaluation during endoscopic ultrasound-guided biopsies of \\ pancreatic solid lesions
}

\author{
Rodrigo Soto-Solis ${ }^{1,2 *}$, Mario Pineda-De Paz', Miriam I. Torres-Ruiz³, Fredy Chablé-Montero ${ }^{4}$, \\ Placido Espinosa-Rosas ${ }^{1}$ y Luis A. Waller-González \\ ${ }^{1}$ Servicio de Endoscopia, Centro Médico Nacional 20 de Noviembre, Instituto de Seguridad y Servicios Sociales de los Trabajadores del Estado; \\ ${ }^{2}$ Departamento de Gastroenterología, Hospital Ángeles Pedregal; ${ }^{3}$ Servicio de Gastroenterología y Endoscopia, Centro Médico Naval, Secretaría \\ de Marina; ${ }^{4}$ Departamento de Patología, Hospital San Ángel Inn Universidad. Ciudad de México, México
}

\section{Resumen}

Antecedentes: La biopsia con aguja fina guiada por ultrasonido endoscópico (BAF-USE) es segura y eficaz para el diagnóstico del adenocarcinoma pancreático. La revisión citológica rápida en sala (ROSE, rapid on-site evaluation) puede mejorar la calidad de la muestra y el rendimiento diagnóstico; en años recientes se ha cuestionado su utilidad. Objetivo: Determinar la eficacia diagnóstica de la ROSE durante las BAF-USE de lesiones pancreáticas sólidas en un nuevo centro de ecoendoscopia. Método: Estudio transversal y comparativo en el que se incluyeron todos los pacientes a quienes se realizó BAF-USE de lesiones pancreáticas sólidas entre enero y julio de 2017. Se evaluaron datos demográficos, ecográficos y de patología de las BAF-USE. Resultados: Se analizaron 23 procedimientos. La mediana de edad fue de 59 años (rango: 46-77). En el grupo con ROSE hubo 13 pacientes (56.5\%) y en el grupo sin ROSE hubo 10 (43.5\%). En el grupo con ROSE, el reporte de patología mostró muestra adecuada en el 100\% (13/13 vs. 5/10; $p=0.007)$, así como diagnóstico de malignidad en el 84.6\% (11/13 vs. 2/10; $p=0.003$ ). Conclusiones: La ROSE es una herramienta útil para mejorar el diagnóstico de BAF-USE de lesiones pancreáticas sólidas, principalmente cuando hay bajo rendimiento diagnóstico y en nuevas unidades de ecoendoscopia.

Palabras clave: Ultrasonido endoscópico. Neoplasias sólidas del páncreas. Biopsia con aguja fina. Revisión citológica en sala.

\begin{abstract}
Background: Endoscopic ultrasound-guided fine needle biopsy (EUS-FNB) is safe and effective for the diagnosis of pancreatic adenocarcinoma. Although rapid on-site evaluation (ROSE) can improve tissue collection and increase diagnostic yield, its utility has been recently questioned. Objective: Determine the diagnostic efficacy of EUS-FNB with ROSE of pancreatic masses in a new echoendoscopy unit. Method: Cross-sectional and comparative study of patients who underwent EUS-FNB of pancreatic masses between January and July 2017. Patient demographics, ultrasonographic details and pathology reports were examined. Results: A total of 23 procedures were analyzed. Median age was 59 years (range: 46-77). The group with
\end{abstract}

\footnotetext{
Correspondencia:

*Rodrigo Soto-Solis

Torre Ángeles, Consultorio 650

Camino a Santa Teresa, 1055

Col. Héroes de Padierna, Del. Magdalena Contreras

C.P. 10700, Ciudad de México, México

Fecha de recepción: 10-09-2019

Cir Cir. 2020;88(4):435-440

E-mail: rodrigosotomd@ hotmail.com

Fecha de aceptación: 08-01-2020

DOI: 10.24875/CIRU.20001572

Contents available at PubMed

www.cirugiaycirujanos.com

0009-7411/@ 2020 Academia Mexicana de Cirugía. Publicado por Permanyer. Este es un artículo open access bajo la licencia CC BY-NC-ND (http://creativecommons.org/licenses/by-nc-nd/4.0/).
} 
ROSE had 13 patients (56.5\%) and the group without ROSE 10 (43.5\%). The final pathology report showed enough and adequate sample in $100 \%$ of the group with ROSE (13/13 vs. $5 / 10 ; p=0.007)$. Diagnosis of malignancy was established in $84.6 \%$ of the biopsies (11/13 vs. 2/10; $p=0.003)$ in the group with ROSE. Conclusions: ROSE is useful to improve the diagnostic efficacy of EUS-FNB of pancreatic masses, especially in new EUS centers or in centers with a low diagnostic yield.

Key words: Endoscopic ultrasound. Pancreatic neoplasms. Fine-needle biopsy. Rapid on-site cytopathologic evaluation.

\section{Introducción}

El cáncer de páncreas es la séptima causa de muerte por cáncer en el mundo. La supervivencia a 5 años es menor del $9 \%{ }^{1}$. Se estima que aproximadamente el $20 \%$ son resecables con fines potencialmente curativos ${ }^{2}$. Por lo tanto, el diagnóstico temprano es crucial para ofrecer al paciente el tratamiento adecuado y mejorar su pronóstico.

Desde su introducción en 1992, la biopsia con aguja fina (con o sin aspiración) guiada por ultrasonido endoscópico (BAF-USE) se ha convertido en una herramienta indispensable en el estudio de las lesiones adyacentes al tracto gastrointestinal, así como en el estudio de las lesiones gastrointestinales subepiteliales $^{3}$. Es una técnica segura, poco invasiva, efectiva y altamente precisa, en especial para lesiones sólidas del páncreas ${ }^{4-7}$. Las complicaciones asociadas a este procedimiento son bajas y en general se reportan en el $0-2 \%$ de los casos $^{8,9}$. Su limitación más importante es que el espécimen citológico puede tener escasa celularidad y falta de arquitectura histológica ${ }^{4}$.

La evaluación citológica rápida en sala (ROSE, rapid on-site evaluation) consiste en la revisión inmediata de las muestras obtenidas por BAF-USE, en términos de suficiencia y diagnóstico preliminar ${ }^{10,11}$. Actualmente la evidencia es controversial, pues la BAF-USE se ha consolidado como una técnica con alto rendimiento diagnóstico (85-87\%), alta sensibilidad (94-96\%) y alta especificidad (95-100\%), aun sin la adición de ROSE $\mathrm{R}^{3,6,7,12}$. Más allá de esto, las Ilamadas agujas histológicas de segunda generación han mostrado resultados promisorios en cuanto a suficiencia, estructura celular y rendimiento diagnóstico $0^{13,14}$.

Alcanzar un alto rendimiento diagnóstico requiere sobrepasar múltiples pasos limitantes, principalmente la experiencia del endosonografista, las características de la aguja, la técnica de adquisición de tejido y la experiencia del patólogo ${ }^{15}$. Por todo esto, la disponibilidad de ROSE podría mejorar la toma de muestra por BAFUSE de lesiones sólidas del páncreas en centros en los que se está iniciando con el ultrasonido endoscópico, en aquellos donde hay médicos en entrenamiento y en aquellos con bajo volumen. El objetivo de este estudio fue comparar la eficacia diagnóstica de la ROSE durante las BAF-USE de lesiones pancreáticas sólidas como parte de la experiencia inicial en un centro de referencia nacional en México.

\section{Método}

Se realizó un estudio retrolectivo y comparativo. Se incluyeron todos los pacientes de ambos sexos, mayores de 18 años, con indicación de BAF-USE por presentar lesiones sólidas del páncreas, en el período de enero a julio de 2017, que fueron atendidos en el servicio de endoscopia del Centro Médico Nacional 20 de Noviembre. El estudio fue aprobado por el comité de ética institucional y todos los pacientes dieron su consentimiento informado por escrito antes del procedimiento. Las biopsias fueron tomadas por dos endosonografistas y la sedación fue asistida por el servicio de anestesiología.

Los resultados de las biopsias fueron divididos en dos grupos: un grupo con ROSE, en el cual se realizó una revisión citológica en la sala de endoscopia hasta considerar que la muestra fue adecuada y posteriormente se envió al servicio de patología para el análisis definitivo, y otro grupo sin ROSE, en el cual las biopsias fueron preservadas en formol al $10 \%$ y enviadas al servicio de patología.

\section{Técnica}

Las BAF-USE fueron tomadas con aguja 22G, sin aspiración, con estilete y retirada lenta; se dieron al menos dos pases con cinco entradas en cuatro direcciones con movimiento en abanico (Figs. 1 y 2). Se utilizó un ecoendoscopio lineal EG-530UT acoplado a la consola SU-8000 (Fujifilm Corporation, Minato-Ku, Tokio, Japón).

\section{Definiciones}

- Muestra adecuada: cuando un patólogo analizaba la muestra y confirmaba la presencia de células glandulares o ductales suficientes para 


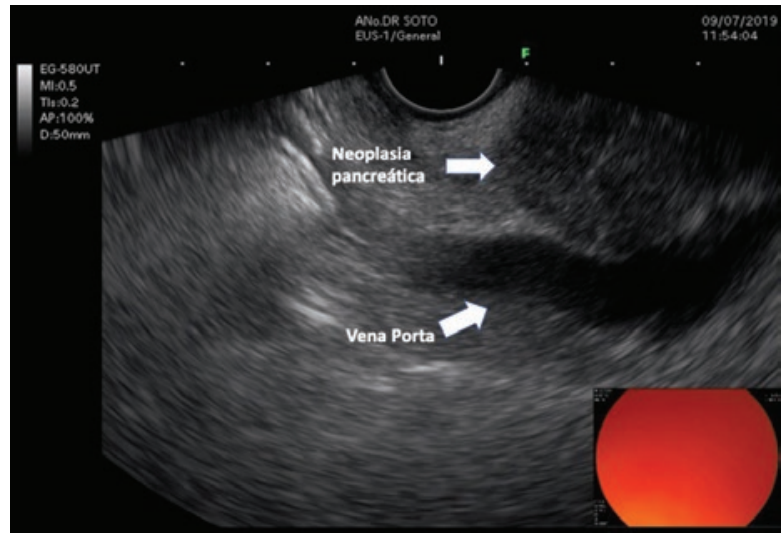

Figura 1. Imagen ecoendoscópica de adenocarcinoma pancreático.

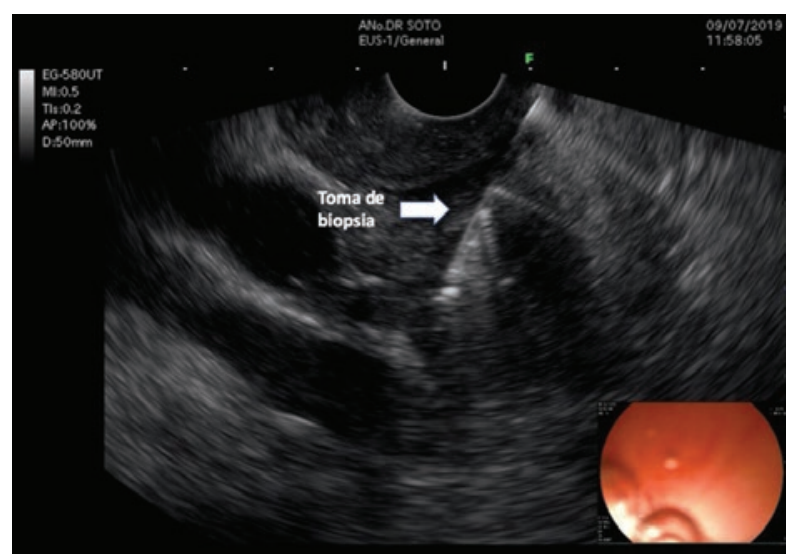

Figura 2. Imagen ecoendoscópica de biopsia con aguja fina de adenocarcinoma pancreático.

poder hacer un diagnóstico histopatológico. En el grupo con ROSE, si la muestra analizada en la sala de endoscopia no era adecuada se tomaba otro pase hasta confirmar que fuera adecuada (Figs. 3 y 4 ).

- Muestra inadecuada: se definió cuando hubo acelularidad, necrosis, ausencia de células glandulares o ductales, o material insuficiente para diagnóstico, y cuando fue reportada textualmente como inadecuada.

- Diagnóstico final: cuando se dio el reporte definitivo en el servicio de patología para descartar o confirmar la presencia de atipia o malignidad.

\section{Análisis estadístico}

Para evaluar los resultados se utilizó estadística descriptiva mediante frecuencias, porcentajes, medianas y rangos. Las diferencias entre los grupos se

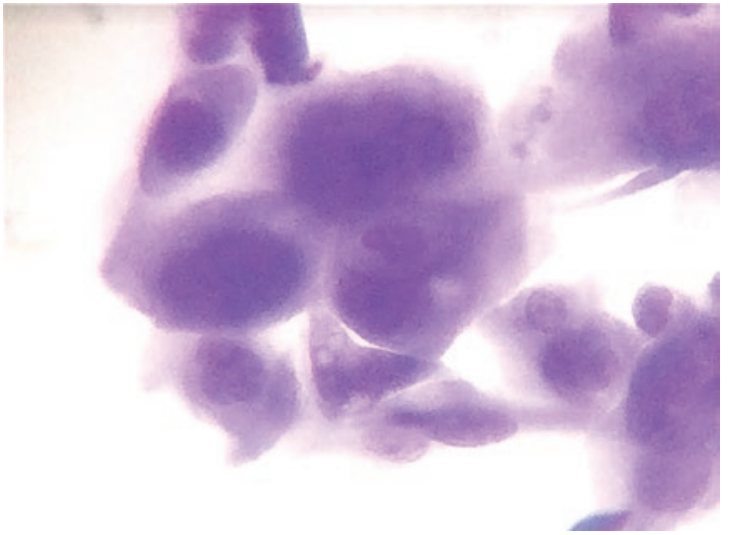

Figura 3. Imagen representativa de la revisión citopatológica en sala (tinción con azul de toluidina).

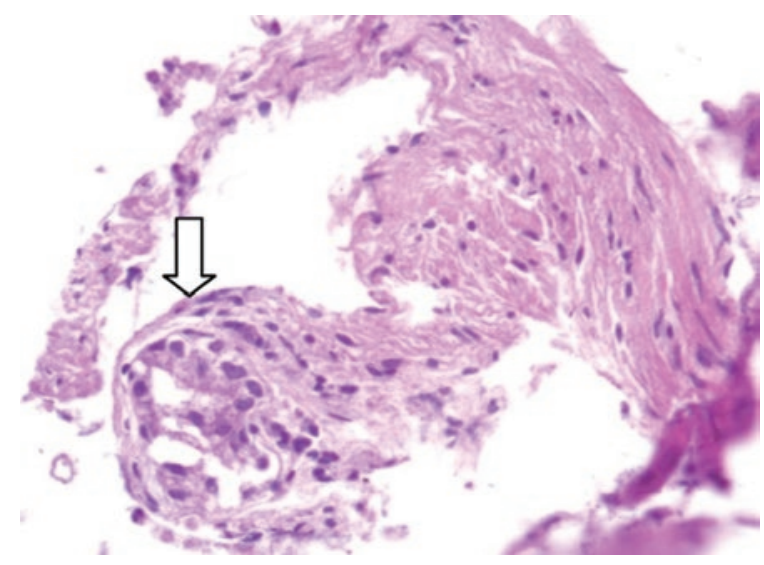

Figura 4. Adenocarcinoma ductal moderadamente diferenciado obtenido por biopsia con aguja fina guiada por ecoendoscopia (bloque celular, tinción con hematoxilina y eosina).

analizaron mediante las pruebas de chi al cuadrado o exacta de Fisher para variables categóricas y t de Student o U de Mann Whitney para variables continuas, según fuera lo apropiado. El análisis estadístico se realizó con SPSS (versión 21, IBM Corp., Armonk, Nueva York, EUA).

\section{Resultados}

Se incluyeron 23 pacientes, la mediana de edad fue de 59 años (rango: 46-77) y 12 (52.2\%) fueron del sexo femenino. En 10 pacientes (43.5\%) se realizó BAF-USE sin evaluación en la sala de endoscopia (grupo sin ROSE) y en 13 pacientes (56.5\%) se realizó evaluación citológica rápida en sala (grupo con ROSE). En la tabla 1 se muestran las principales características de la población, así como la localización y el tamaño de las lesiones pancreáticas. 
Tabla 1. Características generales de los pacientes estudiados

\begin{tabular}{|c|c|c|c|}
\hline & Grupo con ROSE ( $n=13$ ) (\% o rango) & Grupo sin ROSE ( $n=10)$ (\% o rango) & $\mathrm{p}$ \\
\hline Edad, años & $60(47-77)$ & $58(46-72)$ & 0.39 \\
\hline Sexo femenino & $6(46.2)$ & $6(60)$ & 0.68 \\
\hline $\begin{array}{l}\text { Localización de la lesión } \\
\text { Cabeza del páncreas } \\
\text { Proceso uncinado } \\
\text { Cuerpo del páncreas } \\
\text { Cola del páncreas }\end{array}$ & $\begin{array}{c}8(61) \\
3(23.1) \\
1(7.7) \\
1(7.7)\end{array}$ & $\begin{array}{l}7(70) \\
1(10) \\
1(10) \\
1(10)\end{array}$ & 0.87 \\
\hline $\begin{array}{l}\text { Tamaño de la lesión } \\
<30 \mathrm{~mm} \\
\geq 30 \mathrm{~mm} \\
\text { Pases durante BAF-USE }\end{array}$ & $\begin{array}{c}8(61.5) \\
5(38.5) \\
2(2-3)\end{array}$ & $\begin{array}{l}3(30) \\
7(70) \\
3(3-4)\end{array}$ & 0.22 \\
\hline
\end{tabular}

BAF-USE: biopsia con aguja fina guiada por ultrasonido endoscópico; ROSE: rapid on-site evaluation.

Tabla 2. Diagnóstico histológico de las lesiones en todos los pacientes estudiados

\begin{tabular}{lcc}
\hline Tipo de lesión & $\begin{array}{c}\text { Frecuencia } \\
\text { absoluta }(\mathbf{n})\end{array}$ & $\begin{array}{c}\text { Frecuencia } \\
\text { relativa (\%) }\end{array}$ \\
\hline Adenocarcinoma pancreático & 8 & 34.8 \\
Atipia celular, no concluyente & 5 & 21.7 \\
Tumor neuroendocrino & 3 & 13.1 \\
Lesiones benignas & 2 & 8.7 \\
Material insuficiente & 5 & 21.7 \\
Total & 23 & 100 \\
\hline
\end{tabular}

Tabla 3. Muestra considerada adecuada y diagnóstico definitivo en el reporte final de histopatología

\begin{tabular}{|c|c|c|c|}
\hline & $\begin{array}{l}\text { Grupo con ROSE } \\
\qquad(n=13) \\
(56.5 \%)(\%)\end{array}$ & $\begin{array}{c}\text { Grupo sin ROSE } \\
\quad(n=10) \\
(43.5 \%)(\%)\end{array}$ & $p$ \\
\hline $\begin{array}{l}\text { Muestra adecuada } \\
\text { y suficiente }\end{array}$ & $13(100)$ & $5(50)$ & 0.007 \\
\hline $\begin{array}{l}\text { Diagnóstico de } \\
\text { malignidad }\end{array}$ & $11(84)$ & $2(20)$ & 0.003 \\
\hline
\end{tabular}

En la tabla 2 se detallan los diagnósticos por citología para las 23 lesiones estudiadas a partir de las BAF-USE. En el grupo sin ROSE, cinco muestras (50\%) fueron inadecuadas, mientras que en el grupo con ROSE las 13 muestras (100\%) fueron adecuadas, con una diferencia significativa $(p=0.007)$. El número de pases realizados fue menor en el grupo con ROSE, sin haber diferencias significativas al compararlo con el grupo sin ROSE (2 vs. 3; $p=0.21$ ). Del grupo sin ROSE, solo un paciente (10\%) tuvo diagnóstico de malignidad, en comparación con 10 pacientes (76.9\%) en el grupo con ROSE ( $p=0.003$ ) (Tabla 3$)$.

La tasa diagnóstica de la BAF-USE en el grupo sin ROSE fue del $20 \%$, en comparación con el $84.6 \%$ en el grupo con ROSE $(p=0.003)$.

\section{Discusión}

EI USE es la técnica más útil para el diagnóstico del cáncer de páncreas ${ }^{16}$, con un rendimiento global superior al 83\%3,7,17. En México, aún son pocos los hospitales que cuentan con esta herramienta. En nuestro centro se encuentra disponible desde el año 2015 , lo cual ha sido un verdadero reto, pues crear un centro de referencia ha requerido la capacitación del personal, la divulgación de su utilidad y el apoyo de los servicios involucrados. Al analizar nuestros resultados encontramos un gran número de citologías reportadas como negativas o sin muestra suficiente. Este fue el motor para invitar a los patólogos a evaluar algunos casos en nuestra sala.

La ROSE ha demostrado ser útil en múltiples estudios $^{11}$. En el metaanálisis realizado por Hébert-Magee, et al. ${ }^{18}$ se incluyeron 34 estudios con un total de 3,644 pacientes y se encontró una sensibilidad global del 88.6\% para el diagnóstico de adenocarcinoma pancreático, con una especificidad del $99.3 \%$. La ROSE se mantuvo como un determinante del rendimiento diagnóstico de la BAF-USE. Por otro lado, en el metaanálisis y revisión sistemática de Kong, et al. ${ }^{19}$ se evaluaron 1299 pacientes y no se encontraron diferencias cuando se añadía ROSE a la evaluación de 
biopsias pancreáticas y de ganglios linfáticos. Estas controversias en la evidencia hacen difícil saber el rol actual de la ROSE, por lo que algunos expertos consideran que es un «lujo útil y deseable»², aunque no indispensable. Adicionalmente, de forma reciente se han incorporado las agujas de corte histológico de segunda generación. Estas agujas no fueron utilizadas en el presente estudio, pero vale la pena mencionar el metaanálisis del grupo de Todd Baron et al., ${ }^{13}$ en el que se comparó la utilidad de las agujas tradicionales con las histológicas de segunda generación, en escenarios con ROSE y sin ella. Los autores concluyeron que utilizar agujas histológicas de segunda generación resulta equiparable a utilizar una aguja tradicional y tener ROSE ${ }^{13}$. Con estas contradicciones en la evidencia, es difícil saber la utilidad real de la ROSE, pues esto refleja la complejidad de un procedimiento en el que el éxito depende de factores relacionados con el endoscopista, el tipo de aguja, la técnica empleada, el número de pases, el procesamiento de la muestra y la experiencia del patólogo o citotecnólogo3,20,21.

En nuestra experiencia inicial, el uso de ROSE resultó en un mayor número de muestras adecuadas, un mayor rendimiento diagnóstico y un mayor número de diagnósticos definitivos de malignidad (Tablas 2 y 3). Una de las limitantes de nuestro estudio fue el tamaño de la muestra; sin embargo, es un centro que recientemente ha incorporado al ultrasonido endoscópico, por lo que consideramos que puede representar la realidad de muchos centros que buscan añadir esta tecnología a sus unidades de endoscopia. También creemos que el haber invitado al servicio de patología a la sala de endoscopia fue un paso importante para mejorar la participación multidisciplinaria de forma más directa en el abordaje de pacientes con lesiones pancreáticas sólidas. Este cambio impactó en la forma en que ellos evalúan estos casos, pues les hace entender el gran esfuerzo que representa obtener estas muestras. Esta es la importancia real que encontramos en nuestro trabajo: exponer nuestra experiencia a otros centros en donde la tasa diagnóstica de las biopsias por ultrasonido endoscópico es aún baja.

\section{Conclusiones}

La ROSE en las BAF-USE mejora el rendimiento diagnóstico en las lesiones sólidas del páncreas. Consideramos que es una herramienta útil en centros con bajo rendimiento diagnóstico.

\section{Agradecimientos}

A todo el personal del servicio de endoscopia del Centro Médico Nacional 20 de Noviembre y en particular al cuerpo de enfermería, así como al servicio de patología.

\section{Conflicto de intereses}

Los autores declaran no tener conflictos de intereses para la realización de este trabajo.

\section{Responsabilidades éticas}

Protección de personas y animales. Los autores declaran que para esta investigación no se han realizado experimentos en seres humanos ni en animales.

Confidencialidad de los datos. Los autores declaran que han seguido los protocolos de su centro de trabajo sobre la publicación de datos de pacientes.

Derecho a la privacidad y consentimiento informado. Los autores han obtenido el consentimiento informado de los pacientes y/o sujetos referidos en el artículo. Este documento obra en poder del autor de correspondencia.

\section{Bibliografía}

1. Rawla P, Sunkara T, Gaduputi V. Epidemiology of pancreatic cancer: global trends, etiology and risk factors. World J Oncol. 2019;10:10-27.

2. Kedia $P$, Gaidhane M, Kahaleh M. Technical advances in endoscopic ultrasound (EUS)-guided tissue acquisition for pancreatic cancers: how can we get the best results with EUS-guided fine needle aspiration? Clin Endosc. 2013;46:552-62.

3. Chen VK, Eloubeidi MA. Endoscopic ultrasound-guided fine-needle aspiration of intramural and extraintestinal mass lesions: diagnostic accuracy, complication assessment, and impact on management. Endoscopy. 2005:37:984-9.

4. Eloubeidi MA, Jhala D, Chhieng DC, Chen VK, Eltoum I, Vickers S, et al. Yield of endoscopic ultrasound-guided fine-needle aspiration biopsy in patients with suspected pancreatic carcinoma. Emphasis on atypical, suspicious, and false-negative aspirates. Cancer. 2003;99:285-92.

5. Ramírez-Luna MA, Zepeda-Gómez S, Chávez-Tapia NC, Téllez-Ávila FI. Diagnostic yield and therapeutic impact of fine-needle aspiration biopsies guided by endoscopic ultrasound in pancreatic lesions. Rev Invest Clin. 2008;60:11-4.

6. Yang Y, Li L, Qu C, Liang S, Zeng B, Lou Z. Endoscopic ultrasound-guided fine needle core biopsy for the diagnosis of pancreatic malignant lesions: a systematic review and meta-analysis. Sci Rep. 2016;6:22978.

7. Hewitt M, McPhail M, Possamai L, Dhar A, Vlavianos P, Monahan KJ. EUS-guided FNA for diagnosis of solid pancreatic neoplasms: a meta-analysis. Gastrointest Endosc. 2012;75:319-31.

8. Adler D, Jacobson B, Davila R, Hirota WK, Leighton JA, Qureshi WA, et al. ASGE guideline: complications of EUS. Gastrointest Endosc. 2005;61:8-12. Erratum in: Gastrointest Endosc. 2005;61:502.

9. Volmar K, Vollmer R, Jowell P, Nelson RC, Xie HB. Pancreatic FNA in 1000 cases: a comparison of imaging modalities. Gastrointest Endosc. 2005:61:854-61.

10. Iglesias-García J, Domínguez-Muñoz JE, Abdulkader I, Larino-Noia J, Eugenyeva E, Lozano-Leon A, et al. Influence of on-site cytopathology evaluation on the diagnostic accuracy of endoscopic ultrasound-guided fine needle aspiration (EUS-FNA) of solid pancreatic masses. Am J Gastroenterol. 2011;106:1705-10. 
11. Wiersema MJ, Kochman ML, Cramer HM, Tao LC, Wiersema LM. Endosonography-guided real-time fine-needle aspiration biopsy. Gastrointest Endosc. 1994;40:700-7.

12. Bang JY, Kirtane S, Krall K, Navaneethan U, Hasan M, Hawes R, et al. In memoriam: fine-needle aspiration, birth: fine-needle biopsy: the changing trend in endoscopic ultrasound-guided tissue acquisition. Dig Endosc. 2019;31:197-202.

13. Khan MA, Grimm IS, Ali B, Nollan R, Trombazzi C, Ismail MK, et al. A meta-analysis of endoscopic ultrasound-fine-needle aspiration compared to endoscopic ultrasound-fine-needle biopsy: diagnostic yield and the value of onsite cytopathological assessment. Endosc Int Open. 2017;5:E363-75.

14. Duarte-Medrano GA, Téllez-Ávila FI. Rendimiento diagnóstico de la biopsia con aguja ProCore guiada por ultrasonido endoscópico en pacientes con una primera USE-BAAF no diagnóstica y lesiones de difícil diagnóstico por USE-BAAF. Endoscopia. 2013;25:100-4.

15. Matynia AP, Schmidt RL, Barraza G, Layfield LJ, Siddiqui AA, Adler DG, et al. Impact of rapid on-site evaluation on the adequacy of endoscopic-ultrasound guided fine-needle aspiration of solid pancreatic lesions: a systematic review and meta-analysis. J Gastroenterol Hepatol. 2014;29:697-705
16. Lariño Noia J. Últimos avances sobre los tumores pancreáticos. Gastroenterol Hepatol. 2014;37:98-106.

17. Gozalo-Marín J, Vila JJ, Pérez-Miranda M. Role of endoscopic ultrasound in the diagnosis of pancreatic cancer. World $\mathrm{J}$ Gastrointest Oncol. 2014;6:360-8.

18. Hébert-Magee $S$, Bae S, Varadarajulu S, Ramesh J, Frost AR, Eloubeidi MA, et al. The presence of a cytopathologist increases the diagnostic accuracy of endoscopic ultrasound-guided fine needle aspiration cytology for pancreatic adenocarcinoma: a metaanalysis. Cytopathology. 2013;24:159-71.

19. Kong F, Zhu J, Kong X, Sun T, Deng X, Du Y, et al. Rapid on-site evaluation does not improve endoscopic ultrasound-guided fine needle aspiration adequacy in pancreatic masses: a meta-analysis and systematic review. PLoS One. 2016;11:1-14.

20. LeBlanc J Ciaccia D, Al-Assi M, McGrath K, Imperiale T, Tao LC, et al. Optimal number of EUS-guided fine needle passes needed to obtain a correct diagnosis. Gastrointest Endosc. 2004;59:475-81.

21. Lee Y, Moon J, Kim H, Choi HJ, Choi MH, Kim DC, et al. Core biopsy needle versus standard aspiration needle for endoscopic ultrasound-guided sampling of solid pancreatic masses: a randomized parallel-group study. Endoscopy. 2014;46:1056-62. 